\title{
Variação Radial das Estruturas da Madeira de Acrocarpus fraxinifolius Wight \& Arn.
}

\author{
Silvana Nisgoski ${ }^{1}$, Rosilani Trianoski ${ }^{1}$, Graciela Inés Bolzon de Muñiz ${ }^{1}$, \\ Jorge Luis Monteiro de Matos ${ }^{1}$, Marina Stygar ${ }^{2}$ \\ ${ }^{1}$ Departamento de Engenharia e Tecnologia Florestal - DETF, Universidade Federal do Paraná - UFPR \\ ${ }^{2}$ Universidade Federal do Paraná - UFPR
}

\begin{abstract}
RESUMO
A madeira de Acrocarpus fraxinifolius, proveniente de plantios experimentais, foi analisada anatomicamente, avaliando-se a existência de variação dos elementos constituintes no sentido medula-casca. Foram utilizadas árvores com 18 anos de idade, plantadas na região de CorupáSC. O diâmetro tangencial médio dos vasos e o comprimento médio das fibras aumentaram no sentido medula-casca. Os valores médios para a largura da fibra, o diâmetro do lume e a espessura da parede da fibra não apresentaram variação estatisticamente significativa.
\end{abstract}

Palavras-chave: Acrocarpus, características anatômicas, xilema.

\section{Radial Variation of Acrocarpus fraxinifolius Wight \& Arn. Wood Structures}

\begin{abstract}
Acrocarpus fraxinifolius wood from experimental plants was anatomically analyzed evaluating the constituent elements variation from pith to bark. Five 18-year old trees planted in Corupá, State of Santa Catarina were studied. The mean tangential diameter of vessel lumina and fiber length increased from pith to bark. The mean values for fiber width, lumen diameter and fiber wall thickness did not show significant statistical variation.
\end{abstract}

Keywords: Acrocarpus, anatomic characteristic, xylem.

\section{INTRODUÇÃO}

O Acrocarpus fraxinifolius Wight \& Arn., pertencente à família Fabaceae, subfamília Caesalpinioideae, tem como sinonímia botânica Acrocarpus combretiflorus Teysm. \& Binn. e é popularmente conhecido por acrocarpo, mundane, árvore de ripa (Lorenzi et al., 2003), australian ash, indian ash, pink cedar, shingle tree (Onyango et al., 2010), árbol guijarra, cedro rojo, decro rosado, fresno, kenya shade tree, lazcar e mundani (Rocas, 2010). É uma espécie nativa da Ásia, das regiões tropicais de alta pluviosidade, ocorrendo em altitudes que variam de 0 a $2.000 \mathrm{~m}$, e pode ser cultivada em climas com precipitação que varia de 500 a $3.000 \mathrm{~mm}$ e temperaturas médias de 14 a $26{ }^{\circ} \mathrm{C}$ (Martínez et al., 2006). O Acrocarpus fraxinifolius apresenta, no Brasil, um incremento médio anual de 14 a $45 \mathrm{~m}^{3} / \mathrm{ha}$ /ano, sendo as regiões mais recomendadas para o seu plantio o Norte do Paraná, o Sudeste e o Centro-Oeste (Carvalho, 1998). A espécie possui potencial econômico para estabelecimento de plantações produtivas ou recomposição da reserva 
legal em pequenas propriedades rurais do Paraná (Lingnau et al., 2007).

O Acrocarpus fraxinifolius produz madeira dura, de cerne avermelhado, utilizada em construção, mobiliário e produção de celulose (Lorenzi et al., 2003). Em função da sua semelhança física com freixo e nogueira, é usada como substituta dessas espécies na construção de casas e na manufatura de mobília e embalagens. Na África, é utilizada como sombreamento nas plantações de café (Rocas, 2010). É uma madeira de fácil processamento e colagem, obtendo-se superfícies com bom acabamento superficial (Honorato et al., 2005).

A massa específica do Acrocarpus fraxinifolius apresenta variações de acordo com a idade, o local e a procedência, podendo-se encontrar valores, segundo a literatura, de 0,44 a $0,68 \mathrm{~g} / \mathrm{cm}^{3}$ (Whitmore \& Otarola, 1976; Simpson, 1996; Carvalho, 1998; Prado et al., 2003; Honorato et al., 2005; Trianoski, 2010).

As variações nas propriedades tecnológicas da madeira são influenciadas diretamente pelas alterações das dimensões e frequências das células, no sentido medula- casca, geradas pelas mudanças progressivas no câmbio vascular (Kopac \& Sali, 2003). A literatura reporta diferente comportamento em relação à variação das dimensões celulares em espécies florestais nativas e de reflorestamentos, sendo observadas tendências de aumento do diâmetro dos vasos (Urbinati et al., 2003; Dünisch et al., 2004; Chagas et al., 2007; Zanon et al., 2008; Lima et al., 2010; Evangelista et al., 2010) e diminuição da frequência de vasos no sentido medula-casca (Chagas et al., 2007, Lima et al., 2010; Evangelista et al., 2010). Em relação aos raios, Longhi et al. (2010) observaram diminuição na frequência dos raios no sentido medula-casca, enquanto Zanon et al. (2008) observaram aumento no número em Croton floribundus. Por outro lado, Longui et al. (2010) não observaram tendências claras na variação da frequência e do comprimento dos elementos de vaso e diâmetro das fibras em Piptadenia gonoacantha.

Para aumentar as informações sobre a espécie e contribuir para o conhecimento das diferenças estruturais do lenho, este trabalho teve como objetivo a caracterização anatômica da madeira de Acrocarpus fraxinifolius plantada na região de Corupá-SC, analisando a existência de variação dos elementos constituintes do lenho no sentido medulacasca.

\section{MATERIAL E MÉTODOS}

Asárvores utilizadas nesteestudosãoprovenientes de um plantio experimental de propriedade da Batisttella Florestas, com aproximadamente 18 anos de idade, localizado em Corupá-SC, situado a uma latitude de $26^{\circ} 23^{\prime} 19,32^{\prime \prime}$ Oeste, a uma longitude $49^{\circ} 16^{\prime} 50,74^{\prime \prime}$ Sul, e altitude de $75 \mathrm{~m}$. O clima na região é subtropical, com temperatura média entre $15{ }^{\circ} \mathrm{C}$ e $25{ }^{\circ} \mathrm{C}$, oscilando entre $0{ }^{\circ} \mathrm{C}$ e $35{ }^{\circ} \mathrm{C}$. A precipitação média anual varia de 1200 a $1600 \mathrm{~mm}$ e há ocorrência de poucas geadas por ano, sendo que as mesmas não possuem muita intensidade.

A amostragem seguiu a metodologia preconizada pela Norma COPANT 458/1972, totalizando cinco árvores. Após a derrubada, foram retirados discos na posição do DAP, sendo estes divididos em três partes para a caracterização anatômica: próximo à medula (M), intermediário (I) e próximo à casca (C). A descrição macroscópica foi efetuada nos discos e baseada nos procedimentos descritos por Muñiz \& Coradin (1991).

Foram confeccionados corpos de prova orientados nos três eixos anatômicos - transversal, radial e tangencial -, os quais foram fervidos em água para amolecimento. Foram retiradas seções anatômicas nos três planos com espessura de $25 \mu \mathrm{m}$, que foram coradas, desidratadas e montadas em lâminas permanentes. Para a mensuração das características individuais das fibras, foi utilizado o processo de maceração de acordo com a técnica de Franklin (1975) modificada, descrita por Kraus \& Arduin (1997), utilizando-se solução de peróxido de hidrogênio e ácido acético 1:1, e permanecendo o material em estufa a $60{ }^{\circ} \mathrm{C}$ por 24 horas. Este foi então lavado com água destilada, corado com safranina e desidratado em série alcoólica ascendente, sendo, após esse processo, montadas as lâminas permanentes. As medições dos elementos celulares seguiram as normas da IAWA (1989) e de Muñiz \& Coradin (1991). 
Para avaliar a diferença das características médias no sentido medula-casca, foi efetuado o teste de Tukey com $95 \%$ de probabilidade.

\section{RESULTADOS E DISCUSSÃO}

Macroscopicamente, a madeira apresenta cerne e alburno distintos, com coloração variando do bege claro ao rosado, e opaca; anéis de crescimento apresentam-se distintos a olho nu, a textura é média e grã é regular. A Figura 1 ilustra o aspecto macroscópico da madeira do Acrocarpus nos planos transversal e tangencial.

Microscopicamente, a madeira apresenta anéis de crescimento distintos, demarcados por faixas de parênquima marginal.

Vasos: de seção arredondada (43,75-122,17$225 \mu \mathrm{m}, \mathrm{s}=37,86)$ e porosidade difusa, com frequência de 2-5,47-16 por $\mathrm{mm}^{2}(\mathrm{~s}=2,24)$. Solitários, geminados e múltiplos de três, em cachos ocasionais (Figura 2a-c). Elementos vasculares de 202-385-667 $\mu \mathrm{m}(\mathrm{s}=100)$ com placa de perfuração simples, apêndices presentes em ambas as extremidades, elementos vasculares de dois tipos curtos e largos, estreitos e compridos (Figura 3ad). Pontoações intervasculares areoladas alternas, pequenas (Figura 4a), ornamentadas (Figura 4b). Pontoações raiovasculares e parênquimo vasculares (Figura $4 \mathrm{c}-\mathrm{d}$ ) semelhantes às intervasculares.

Parênquima axial em faixas marginais, paratraqueal vasicêntrico, aliforme e confluente (Figura 2a,b). Cristais romboédricos presentes (Figura 4e).
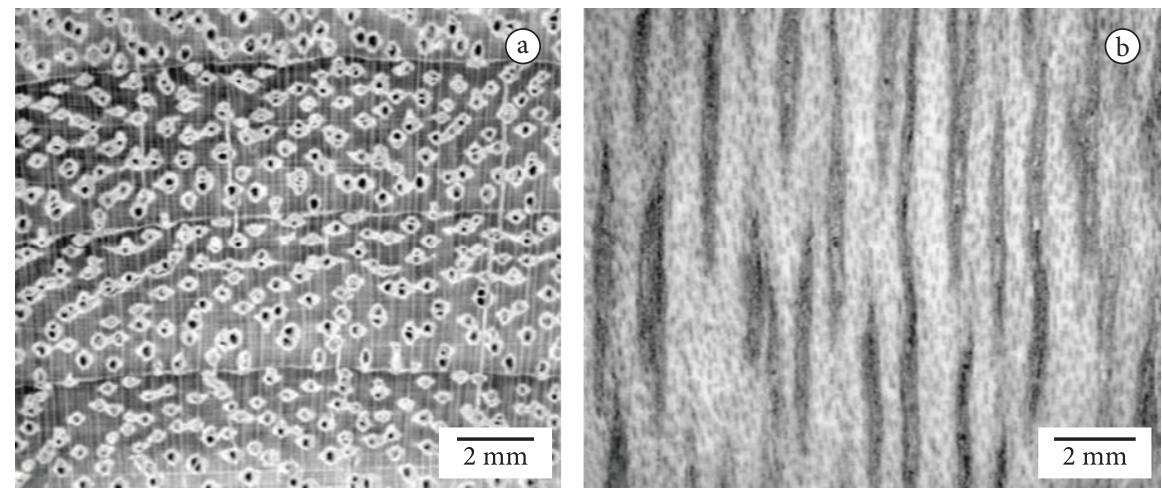

Figura 1. Aspecto macroscópico da madeira de Acrocarpus. a) plano transversal; b) plano tangencial. Figure 1. Macroscopic aspect of Acrocarpus wood. a) transverse section; b) tangential section.
Raios: heterocelulares (Figura 4f) com frequência de 2-5,19-10 por $\mathrm{mm}(\mathrm{s}=1,47)$, predominantemente multisseriados, com 2-4 células de largura e 6-16,74-36 células $(s=5,66)$ e 125-283,7-587,50 $\mu \mathrm{m}$ $(s=92,52)$ de altura. Cristais isolados ocasionalmente mostram-se presentes nas células marginais.

Fibras libriformes. Fibras septadas presentes. Pontoações simples diminutas, curtas (347-1295-2300 $\mu \mathrm{m}, \mathrm{s}=334)$ de paredes delgadas a espessas (1,8-5,7-10 $\mu \mathrm{m}, \mathrm{s}=1,7)$ (Figura 3e,f).

Máculas medulares mostraram-se presentes em algumas amostras (Figura 2d).

As características observadas na madeira de reflorestamento são compatíveis com as descritas por Gamble (1972) e Wheeler (2004), estudando o Acrocarpus na região de origem.

A variação nas dimensões dos elementos celulares é apresentada nas Tabelas 1 a 3, bem como a análise das médias pelo teste de Tukey.

O diâmetro tangencial médio dos vasos aumentou no sentido medula-casca e o número de vasos por milímetro quadrado diminuiu. $\mathrm{O}$ aumento verificado no diâmetro tangencial médio dos vasos e a presença de vasos solitários em grande quantidade são aspectos característicos do desenvolvimento do vegetal, sendo considerados por Carlquist (1989) como uma adaptação anatômica do xilema para incrementar o volume de seiva mineral, aumentando a sua capacidade fotossintética.

O padrão observado de aumento no diâmetro dos vasos no sentido medula-casca também foi relatado em Terminalia ivorensis (Urbinati et al., 

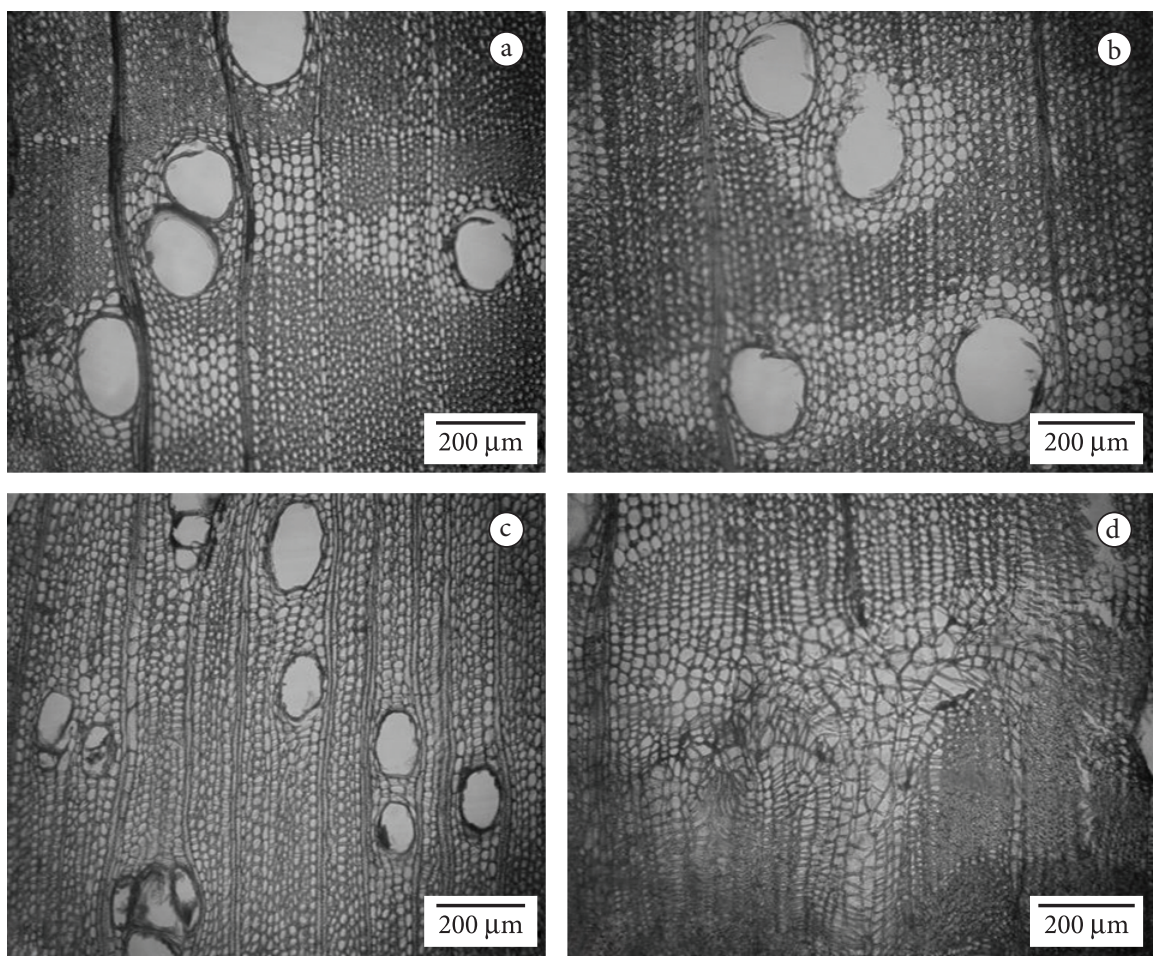

Figura 2. Variação no diâmetro dos vasos e parênquima axial no sentido casca-medula. a) Região próxima à casca; b) Região intermediária; c) Região próxima à medula; d) Mácula medular.

Figure 2. Variation in pore diameter and axial parenchyma from pith to bark. a) Region near bark; b) Intermediate region; c) Region near pith; d) Stain pith.

Tabela 1. Dimensões e frequência dos vasos no sentido medula-casca.

Table 1. Vessel dimension and frequency from pith to bark.

\begin{tabular}{|c|c|c|c|c|}
\hline & Posição & $\begin{array}{l}\text { Diâmetro } \\
(\mu \mathrm{m})\end{array}$ & $\begin{array}{c}\text { Vasos } / \mathbf{m m}^{2} \\
\text { (n) }\end{array}$ & $\begin{array}{c}\text { Comprimento } \\
(\mu \mathrm{m})\end{array}$ \\
\hline Mínimo & $\mathbf{M}$ & 43,75 & 4,00 & 213,18 \\
\hline Média & & $89,40^{c}$ & $7,74^{\mathrm{b}}$ & $325,49^{c}$ \\
\hline Máximo & & 156,25 & 16,00 & 490,24 \\
\hline Desvio & & 19,34 & 2,25 & 71,44 \\
\hline Mínimo & I & 62,50 & 2,00 & 202,95 \\
\hline Média & & $130,58^{b}$ & $4,46^{\mathrm{a}}$ & $387,52^{b}$ \\
\hline Máximo & & 218,75 & 8,00 & 599,27 \\
\hline Desvio & & 31,68 & 1,18 & 77,96 \\
\hline Mínimo & $\mathrm{C}$ & 43,75 & 2,50 & 226,31 \\
\hline Média & & $146,53^{a}$ & $4,22^{\mathrm{a}}$ & $442,35^{a}$ \\
\hline Máximo & & 225,00 & 7,00 & 667,77 \\
\hline Desvio & & 34,54 & 0,96 & 110,08 \\
\hline
\end{tabular}

*Médias seguidas pela mesma letra na coluna não diferem entre si pelo teste de Tukey a $95 \%$ de probabilidade. (M) próximo à medula;

(I) intermediário; (C) próximo à casca.

2003), Ilex paraguariensis (Dünish et al., 2004), Eremanthus erythropappus (Chagas et al., 2007), Croton floribundus (Zanon et al., 2008; Lima et al., 2010), clones de Eucalyptus camaldulensis e
E. urophylla (Evangelista et al., 2010); a diminuição da frequência de vasos foi relatada em estudos de Chagas et al. (2007), Lima et al. (2010); Evangelista et al. (2010). Já Longui et al. (2010) 

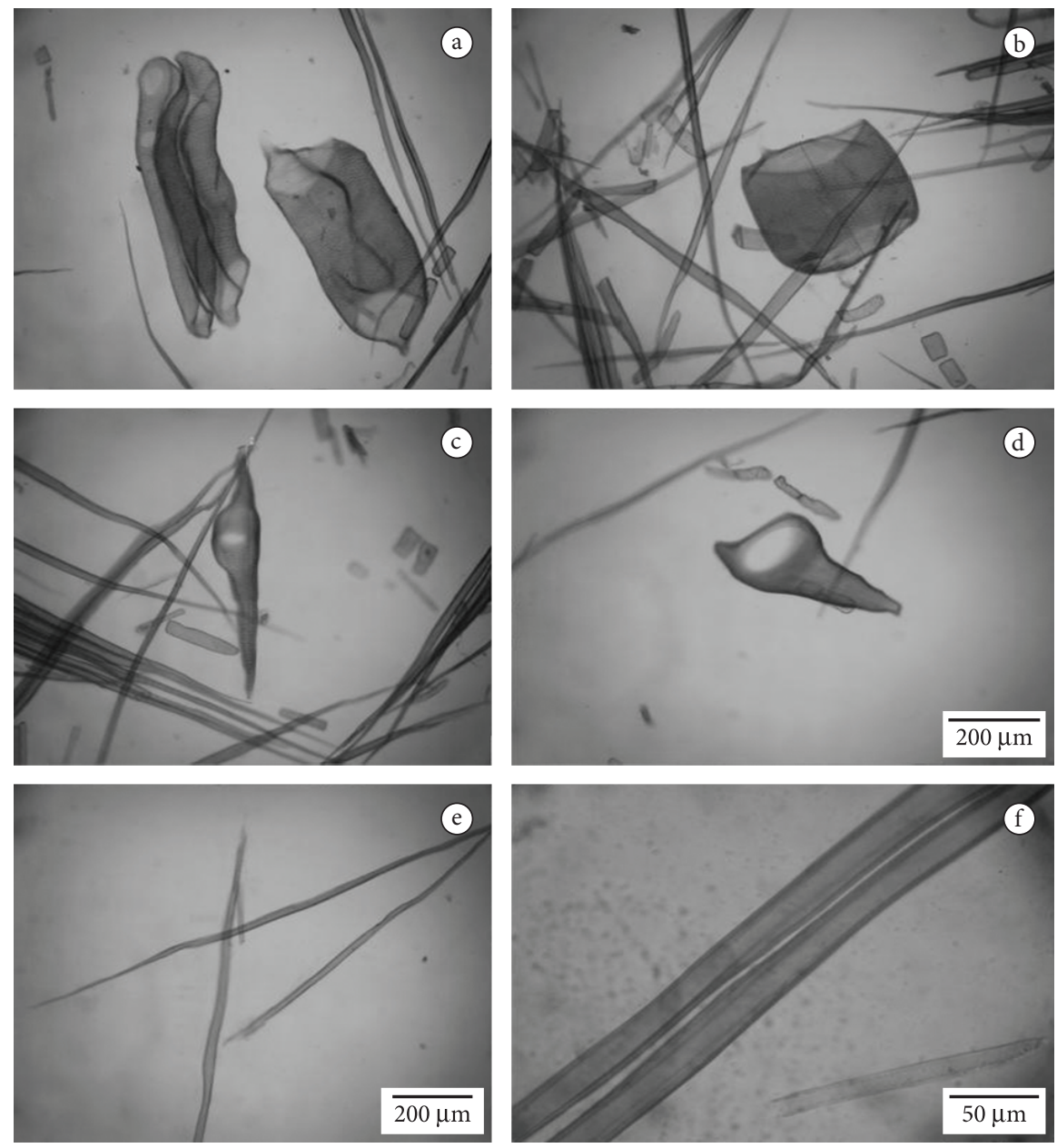

Figura 3. a-d) Variação nas dimensões dos elementos de vaso e apêndices; e-f) Fibras.

Figure 3. a-d) Vessel elements dimension and appendices variation. e-f) fibers.

Tabela 2. Dimensões e frequência dos raios no sentido medula-casca.

Table 2. Rays dimension and frequency from pith to bark.

\begin{tabular}{|c|c|c|c|c|c|c|}
\hline & Posição & $\begin{array}{c}\text { Raio/mm } \\
\text { (n) }\end{array}$ & $\begin{array}{c}\text { Altura } \\
\text { (n) }\end{array}$ & $\begin{array}{l}\text { Largura } \\
\text { (n) }\end{array}$ & $\begin{array}{c}\text { Altura } \\
\text { ( } \mu \mathrm{m})\end{array}$ & $\begin{array}{c}\text { Largura } \\
\quad(\mu \mathrm{m})\end{array}$ \\
\hline Mínimo & $\mathbf{M}$ & 3,00 & 6,00 & 1,00 & 125,00 & 12,50 \\
\hline Média & & $6,44^{\mathrm{b}}$ & $16,71^{\mathrm{a}}$ & $2,58^{b}$ & $287,25^{a}$ & $29,40^{\mathrm{a}}$ \\
\hline Máximo & & 10,00 & 36,00 & 4,00 & 587,50 & 43,75 \\
\hline Desvio & & 1,43 & 5,96 & 0,61 & 103,50 & 7,01 \\
\hline Mínimo & I & 2,00 & 9,00 & 2,00 & 125,00 & 18,75 \\
\hline Média & & $4,54^{\mathrm{a}}$ & $17,08^{\mathrm{a}}$ & $2,85^{\mathrm{a}}$ & $279,25^{a}$ & $29,20^{\mathrm{a}}$ \\
\hline Máximo & & 7,00 & 33,00 & 4,00 & 556,25 & 56,25 \\
\hline Desvio & & 0,97 & 5,99 & 0,61 & 92,52 & 6,09 \\
\hline Mínimo & C & 2,00 & 7,00 & 2,00 & 150,00 & 18,75 \\
\hline Média & & $4,59^{\mathrm{a}}$ & $16,42^{\mathrm{a}}$ & $2,98^{a}$ & $283,30^{a}$ & $30,85^{a}$ \\
\hline Máximo & & 7,00 & 35,00 & 4,00 & 562,50 & 50,00 \\
\hline Desvio & & 1,08 & 5,01 & 0,54 & 80,70 & 6,48 \\
\hline
\end{tabular}

*Médias seguidas pela mesma letra na coluna não diferem entre si pelo teste de Tukey a 95\% de probabilidade; (M) próximo à medula;

(I) intermediário; (C) próximo à casca. 

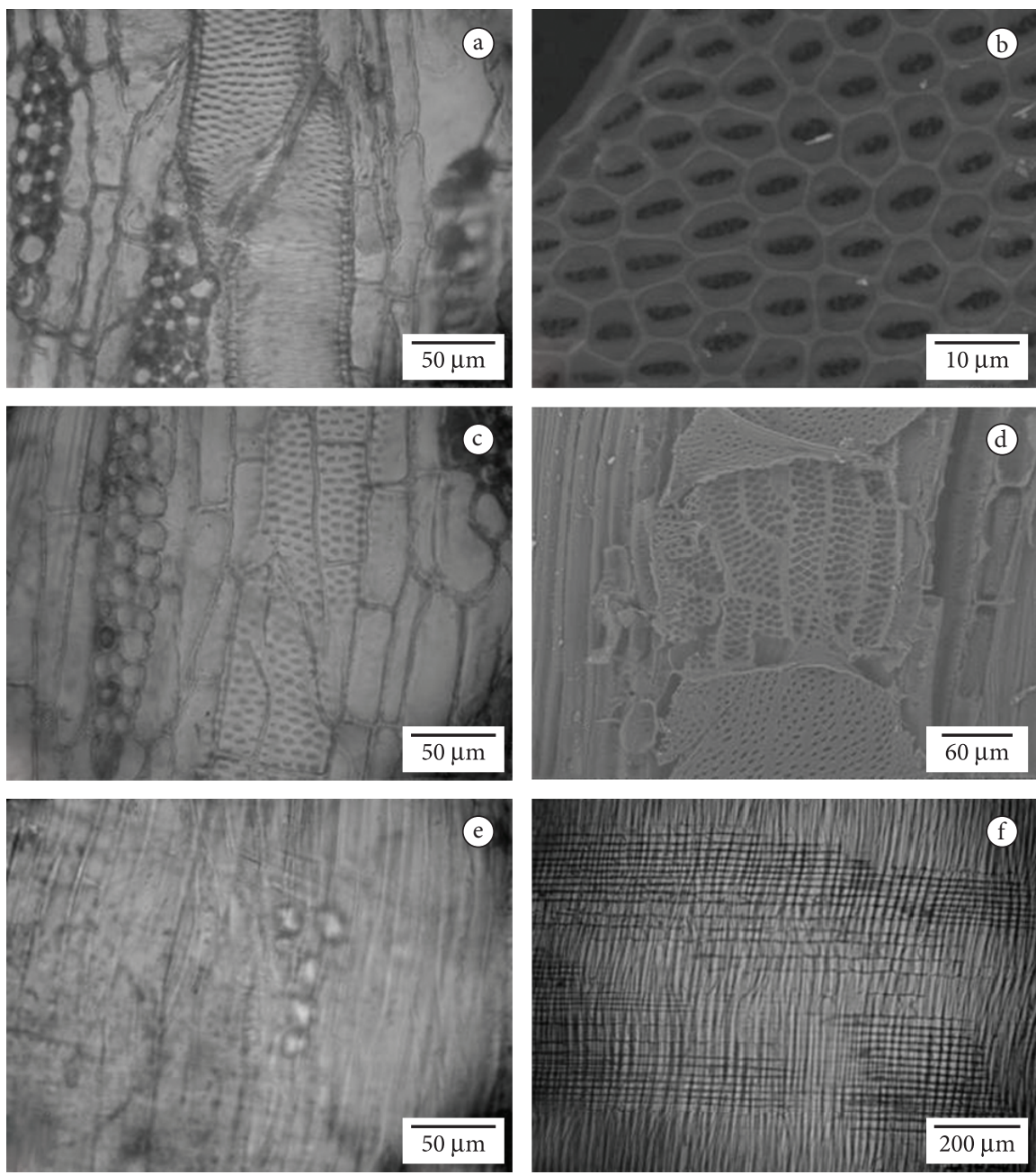

Figura 4. a) Pontoações intervasculares; b) Pontoações parenquimovasculares. c) Cristais romboédricos; d) Raios heterogêneos.

Figure 4. a) Intervessel pits; b) Parenchyma-vessel pitting; c) Prismatic crystals; d) Heterogeneous rays.

Tabela 3. Dimensões das fibras no sentido medula-casca.

Table 3. Fiber dimension from pith to bark.

\begin{tabular}{|c|c|c|c|c|c|}
\hline & Posição & $\begin{array}{l}\text { Comprimento } \\
\text { fibra ( } \mu \mathrm{m})\end{array}$ & $\begin{array}{l}\text { Largura fibra } \\
\quad(\mu \mathrm{m})\end{array}$ & $\begin{array}{c}\text { Diâmetro lume } \\
(\mu \mathrm{m})\end{array}$ & $\begin{array}{l}\text { Espessura da } \\
\text { parede ( } \mu \mathrm{m})\end{array}$ \\
\hline Mínimo & M & 395,26 & 15,00 & 10,00 & 1,86 \\
\hline Média & & $1043,44^{\mathrm{b}}$ & $27,97^{\mathrm{a}}$ & $16,82^{\mathrm{a}}$ & $5,57^{\mathrm{a}}$ \\
\hline Máximo & & 1650,00 & 50,00 & 40,00 & 10,00 \\
\hline Desvio & & 224,83 & 5,71 & 5,18 & 1,69 \\
\hline Mínimo & I & 599,38 & 16,94 & 9,47 & 2,21 \\
\hline Média & & $1369,57^{a}$ & $28,79^{a}$ & $17,55^{\mathrm{a}}$ & $5,62^{a}$ \\
\hline Máximo & & 2300 & 41,84 & 30,77 & 10,00 \\
\hline Desvio & & 290,35 & 5,40 & 4,61 & 1,77 \\
\hline Mínimo & $\mathrm{C}$ & 346,73 & 14,83 & 8,28 & 2,13 \\
\hline Média & & $1472,02^{\mathrm{a}}$ & $27,30^{\mathrm{a}}$ & $15,87^{\mathrm{a}}$ & $5,71^{\mathrm{a}}$ \\
\hline Máximo & & 2075 & 40,00 & 30,00 & 10,00 \\
\hline Desvio & & 318,23 & 4,88 & 4,45 & 1,76 \\
\hline
\end{tabular}

${ }^{*}$ Médias seguidas pela mesma letra na coluna não diferem entre si pelo teste de Tukey a 95\% de probabilidade; (M) próximo à medula;

(I) intermediário; (C) próximo à casca. 
não observaram tendências claras na variação da frequência e do comprimento dos elementos de vaso em Piptadenia gonoacantha.

A altura dos raios em número de células e em micrômetros, e a largura em micrômetros não variaram de acordo com a posição. $\mathrm{O}$ número de raios por milímetro foi maior na região próxima à medula Em relação aos raios, Longhi et al. (2010) observaram diminuição na frequência dos raios no sentido medula-casca, enquanto Zanon et al. (2008) observaram aumento no número em Croton floribundus.

As análises das tendências ecológicas relacionadas ao tamanho e ao tipo de raio possuem difícil interpretação nos diferentes sistemas ecológicos (Baas 1982). Alves \& Angyalossy-Alfonso (2000, 2002) determinaram tendências anatômicas para 491 espécies incluídas em 22 famílias, de diferentes ambientes, latitudes e altitudes, e verificaram que a composição dos raios não segue um padrão.

Não foram observadas variações no sentido medula-casca na largura da fibra, no diâmetro do lume e na espessura da parede das fibras de maneira estatisticamente significativas. O comprimento das fibras aumentou no sentido medula-casca. Alves \& Angyalossy-Alfonso (2002) observaram a influência das características do local, como temperatura, umidade e tipo de vegetação na espessura da parede das fibras de 491 espécies brasileiras.

Em comparação com os resultados disponíveis na literatura para a dimensão das fibras, pode-se verificar que os resultados obtidos neste trabalho estão próximos às informações descritas por Whitmore \& Otarola (1976), que relatam um comprimento médio de 1,35 mm e largura de $31 \mu \mathrm{m}$ para a espécie. Estes resultados também se apresentam similares aos valores obtidos por Prado et al. (2003), que, ao avaliarem seis procedências de Acrocarpus fraxinifolius aos oito anos de idade instaladas em um experimento em Foz do Iguaçu-PR, obtiveram comprimento médio $1,20 \mathrm{~mm}$, largura de 25,00 $\mu \mathrm{m}$, diâmetro de lume de $15,00 \mu \mathrm{m}$ e espessura de parede de $4,78 \mu \mathrm{m}$. As pequenas diferenças encontradas entre esses estudos podem ser justificadas por fatores inerentes à procedência, à idade e à região de plantio.

$\mathrm{Na}$ análise das dimensões das fibras, os resultados na literatura também são variáveis, sendo observado aumento no comprimento no sentido radial em trabalhos de Testoni et al. (2009), Andrade et al. (2009), Garcia et al. (2009) e Evangelista et al. (2010), além do aumento na largura das fibras em Teixeira (2008) e Suckow et al. (2009). Note-se que Zanon et al. (2008) não encontraram diferença significativa.

\section{CONCLUSÕES}

A análise da estrutura anatômica da madeira de Acrocarpus fraxinifolius permite as seguintes conclusões:

- A madeira apresentou variação, estatisticamente significativa no nível de 5\% de probabilidade, no diâmetro e no comprimento dos elementos de vaso e no comprimento da fibra no sentido medula-casca, resultado dos diferentes estágios de crescimento da planta;

- As características anatômicas da madeira proveniente de plantios experimentais são semelhantes às da região de ocorrência natural, mantendo-se as propriedades tecnológicas do material; pode, dessa forma, ser utilizada em reflorestamentos no sul do Brasil.

\section{AGRADECIMENTOS}

Os autores agradecem à Battistella Florestas, pela concessão de uso dos plantios experimentais, ao Professor Antonio Higa, Coordenador do Projeto FINEP/BATTISTELA/UFPR, e à Financiadora de Estudos e Projetos - FINEP.

\section{STATUS DA SUBMISSÃO}

Recebido: $05 / 12 / 2011$

Aceito: 14/06/2012

Publicado: 30/09/2012

\section{AUTOR (ES) PARA CORRESPONDÊNCIA}

\section{Silvana Nisgoski}

Departamento de Engenharia e Tecnologia Florestal - DETF, Universidade Federal do Paraná - UFPR, Campus III, Av. Prefeito Lothário Meissner, 900, Jardim Botânico, CEP 80210-170, Curitiba, PR, Brasil e-mail: nisgoski@ufpr.br 


\section{Rosilani Trianoski \\ Departamento de Engenharia e Tecnologia Florestal - DETF, Universidade Federal do Paraná - UFPR, Campus III, Av. Prefeito Lothário Meissner, 900, Jardim Botânico, CEP 80210-170, Curitiba, PR, Brasil e-mail: rosilani@ufpr.br}

\section{REFERÊNCIAS}

Alves ES, Angyalossy-Alfonso V. Ecological trends in the wood anatomy of some Brazilian species. 1. Growth rings and vessels. IAWA Journal 2000; 20(1): 3-30.

Alves ES, Angyalossy-Alfonso V. Ecological trends in the wood anatomy of some Brazilian species. 2. Axial parenchyma, rays e fibers. IAWA Journal 2002; 23(4): 391-418.

Andrade IM, Longui EL, Lima IL, Florsheim SMB. Efeito da procedência na variação radial do comprimento das fibras e densidade básica de Gallesia integrifolia (Spreng) Harms. Instituto Florestal, Série Registros 2009; 40: 39-43.

Baas P. Systematic, phylogenetic and ecological wood anatomy. In: Baas P. New perspectives in wood anatomy: systematic, phylogenetic and ecological wood anatomy. London: Publishers Junk; 1982.

Carlquist S. Comparative wood anatomy: systematic ecological, and evolutionary aspects of dicotyledon wood. Berlin: Springer-Verlag; 1989.

Carvalho PE. Espécies introduzidas alternativas às do gênero Pinus e Eucalyptus para reflorestamento no Centro-sul do Brasil. In: Galvão APM, coordenador. Espécies não tradicionais para plantios com finalidades produtivas e ambientais. Colombo: Embrapa Florestas; 1998.

Chagas MP, Tomazello Filho M, Lisi CS. Caracterização macro e microscópica da madeira de candeia (Eremanthus erythropappus, Asteraceae). Revista Brasileira de Biociências 2007; 5(1): 156-158.

Comissão Panamericana de Normas Técnicas COPANT. COPANT 458: Maderas - Selección y colección de muestras. COPANT; 1972.

Dünisch O, Reissmann $\mathrm{CB}$, Oliszeski AV. Variability of vessel characteristics in xylem of Ilex paraguariensis (mate-tree) from south Brazil. IAWA Journal 2004; 25(4): 449-458.

Evangelista WV, Silva JC, Valle MLA, Xavier BA. Caracterização anatômica quantitativa da madeira de clones de Eucalyptus camaldulensis Dehnh. e Eucalyptus urophylla S.T.Blake. Scientia Forestalis 2010; 38(89): 273-284.
Gamble JS. A manual of Indian timbers. Dehra Dun: Bishen Singh Mahendra Pal Singh; 1972.

Garcia MF, Florsheim SMB, Lima IL, Longui EI. Variação radial da densidade básica e comprimento das fibras de diferentes procedências de Cariniana legalis - Lecythidaceae. Instituto Florestal, Série Registros 2009; 40: 75-80.

Honorato SJA, Parraguirre LJFC, Quintanar OJ, Rodriguez, CHM. Cedro rosado (Acrocarpus fraxinifolius) una opción agroforestal para la sierra Norte del estado de Puebla. INIFAP; 2005. Folleto Técnico, v. $1,41 \mathrm{p}$.

International Aviation Womens Association - IAWA. List of microscopic features for hardwood identification. IAWA Bulletin 1989; 10(3): 219-332.

Kopac J, Sali S. Wood: an important material in manufacturing technology. Journal of Material Processing Technology 2003; 133(1-2): 134-142. http:// dx.doi.org/10.1016/S0924-0136(02)00217-0

Kraus JE, Arduin M. Manual básico de métodos em morfologia vegetal. Seropédica: Editora Universidade Rural; 1997.

Lima IL, Zanon BR, Longui EL, Florsheim SMB. Variação radial da densidade básica e dimensões celulares de Croton floribundus. Floresta 2010; 40(3): 663-670.

Lingnau C, Higa AR, Martines DT, Arce JE, Silva IC. Sistema interativo de informações para a seleção de espécies florestais para reflorestamentos em pequenas propriedades do Paraná. In: Anais do XIII Simpósio Brasileiro de Sensoriamento Remoto; 2007; Florianópolis. Florianópolis: INPE; 2007, p.1719- 1724.

Longui EL, Lima IL, Junior FGS, Bufolo A, Suckow IMS, Florsheim SMB. Variação radial das características anatômicas, densidade aparente, teores de extrativos, lignina e holocelulose na madeira de Piptadenia gonoacantha (Mart.) Macbr. Scientia Forestalis 2010; 38(87): 341-353.

Lorenzi H, Souza HM, Torres MAV, Bacher LB. Árvores exóticas no Brasil: madeireiras, ornamentais e aromáticas. Nova Odessa, SP: Instituto Plantarum, 2003.

Martínez PE, García JMM, Sánchez MLH, Pérez GO. Cultivo intercalado de cedro rosado y su efecto sobre el contenido de materia orgánica en suelo. Revista UDO Agrícola 2006; 6(1): 109-113.

Muñiz GIB, Coradin VR. Normas de procedimentos em estudo de anatomia da Madeira: I - Angiospermae, II- Gimnospermae. Brasília: Laboratório de Produtos Florestais; 1991. (Série Técnica, n. 15).

Onyango G, Ekakoro E, Sang J. Emiti Nibwo Bulora woodlot technical specification. SCC-Vi Agroforestry; 2010. 
Prado CA, Pereira JCD, Mattos PP, Schaitza EG, Higa AR. Características físicas e químicas da madeira de Acrocarpus fraxinifolius Wight \& Arn. Colombo: Embrapa Florestas; 2003. (Boletim de Pesquisa e Desenvolvimento, n. 14).

Rocas AN. Acrocarpus fraxinifolius Wight \& Arn. [on line]. [cited 2010 Ago 6]. Available from: www.rngr.net/ publications.

Simpson WT. Method to estimate dry-kiln schedules and species groupings: Tropical and temperate hardwoods. Madison: U.S. Department of Agriculture, Forest Service, Forest Products Laboratory; 1996. Res. Pap. FPL-RP-548.

Suckow IMS, Longui EL, Lima IL, Florsheim SMB, Aguiar OT. Anatomia da madeira e densidade básica de angico-branco Anadenanthera colubrina (Vell) Brenan. Instituto Florestal, Série Registros 2009; 40: 131-135.

Teixeira BM. Variabilidade radial e longitudinal de propriedades da madeira de angico vermelho (Anadenanthera peregrina (L.) Speg) [tese]. Viçosa: Setor de Ciências Agrárias, Universidade Federal de Viçosa; 2008.
Testoni LN, Longui EL, Lima IL, Florsheim SMB, Melo ACG. Anatomia, densidade aparente e velocidade de propagação do som na madeira de Plathymenia reticulata. Instituto Florestal, Série Registros 2009; 40: 125-130.

Trianoski R. Avaliação do potencial de espécies florestais alternativas, de rápido crescimento, para produção de painéis de madeira aglomerada [dissertação]. Curitiba: Setor de Ciências Agrárias, Universidade Federal do Paraná; 2010.

Urbinati CV, Azevedo AA, Monteiro da Silva EA, Lisboa PLB. Variação estrutural quantitativa no lenho de Terminalia ivorensis A. Chev., Combretaceae. Acta Botânica Brasileira 2003; 17(3): 421-437. http://dx.doi. org/10.1590/S0102-33062003000300009

Wheeler E. InsideWood. [on line]. [cited 2011 Nov 8]. Available from: http://insidewood.lib.ncsu.edu/search.

Whitmore JL, Otarola T. Acrocarpus fraxinifolius Wight, especie de rápido crecimiento inicial, buena forma y madera de usos múltiples. Turrialba 1976; 2: 201-204.

Zanon BR, Longui EI, Florsheim SMB, Lima IL. Variação radial da densidade básica e dimensões celulares de Croton floribundus Spreng. na Serra da Cantareira, SP. Instituto Florestal, Série Registros 2008; 36: 25-29. 